\title{
The prediction of type 2 diabetes in women with previous gestational diabetes mellitus using lipidomics
}

\author{
Martha Lappas ${ }^{1,2}$ - Piyushkumar A. Mundra ${ }^{3}$ - Gerard Wong ${ }^{3} \cdot$ Kevin Huynh $^{3}$. \\ Debra Jinks $^{2} \cdot$ Harry M. Georgiou ${ }^{2,4} \cdot$ Michael Permezel $^{2,4} \cdot$ Peter J. Meikle $^{3,5}$
}

Received: 21 December 2014 / Accepted: 23 March 2015 /Published online: 17 April 2015

(C) Springer-Verlag Berlin Heidelberg 2015

\begin{abstract}
Aims/hypothesis The risk of developing diabetes is greater for women with previous gestational diabetes mellitus (GDM). In the general population, plasma lipidomic analysis can identify individuals at risk of developing type 2 diabetes. The aim of this study was to determine whether circulating lipid levels 12 weeks following a GDM pregnancy were associated with an increased risk of developing type 2 diabetes.

Methods Plasma lipid profiles containing $>300$ lipids were measured in 104 normal glucose-tolerant women 12 weeks following an index GDM pregnancy using electrosprayionisation tandem mass spectrometry. Women were assessed for 10 years for development of overt type 2 diabetes.

Results Among the 104 women with previous GDM, 21 (20\%) developed diabetes during the median follow-up period of 8.5 years. Three lipid species, the cholesteryl ester species CE
\end{abstract}

Electronic supplementary material The online version of this article (doi:10.1007/s00125-015-3587-7) contains peer-reviewed but unedited supplementary material, which is available to authorised users.

Martha Lappas

mlappas@unimelb.edu.au

1 Obstetrics, Nutrition and Endocrinology Group, Department of Obstetrics and Gynaecology, University of Melbourne, Mercy Hospital for Women, Level 4/163 Studley Road,

Heidelberg 3084, VIC, Australia

2 Mercy Perinatal Research Centre, Mercy Hospital for Women, Heidelberg, VIC, Australia

3 Metabolomics Laboratory, Baker IDI Heart and Diabetes Institute, Melbourne, VIC, Australia

4 Department of Obstetrics and Gynaecology, University of Melbourne, Mercy Hospital for Women, Heidelberg, VIC, Australia

5 Department of Biochemistry and Molecular Biology, University of Melbourne, Melbourne, VIC, Australia
20:4, the alkenylphosphatidylethanolamine species PE(P-36:2) and the phosphatidylserine species PS 38:4, were independently and positively associated with the development of type 2 diabetes. In a clinical model of prediction of type 2 diabetes that included age, BMI, and levels of pregnancy fasting glucose, postnatal fasting glucose, triacylglycerol and total cholesterol, the addition of these three lipid species resulted in an improvement in the net reclassification index of $22.3 \%$.

Conclusions/interpretation The lipid species CE 20:4, PE(P-36:2) and PS 38:4 were significant risk factors for the development of type 2 diabetes in women with a previous history of GDM. This report is the first to use plasma lipidomic analysis to identify individual lipids as potential biomarkers for the prediction of type 2 diabetes in women with a history of GDM.

Keywords Gestational diabetes mellitus · Lipids · Type 2 diabetes

\begin{tabular}{ll}
\multicolumn{2}{l}{ Abbreviations } \\
AusDiab & Australian Diabetes, Obesity and Lifestyle \\
CE & Cholesteryl ester species \\
Cer & Ceramide species \\
GDM & Gestational diabetes mellitus \\
IGT & Impaired glucose tolerance \\
NGT & Normal glucose tolerance \\
PC(O) & Alkylphosphatidylcholine species \\
PE(O) & Alkylphosphatidylethanolamine species \\
PE(P) & Alkenylphosphatidylethanolamine species \\
PI & Phosphatidylinositol species \\
PS & Phosphatidylserine species \\
ROC & Receiver operating characteristic \\
SM & Sphingomyelin species
\end{tabular}




\section{Introduction}

The prevalence of diabetes worldwide is increasing. Alarmingly, it is predicted that by 2030 there will be 439 million people living with diabetes [1]. Globally, type 2 diabetes is a major cause of morbidity and mortality $[2,3]$. The cost of managing patients with type 2 diabetes places an enormous burden on the healthcare systems [4]. It is now recognised that gestational diabetes mellitus (GDM) is an important predictor of the development of type 2 diabetes. We have previously reported that the risk of developing diabetes is 9.6 times greater for patients with a previous history of GDM, with a cumulative risk of $25 \% 15$ years after diagnosis [5]. Thus, the accurate identification of women with GDM who are at risk of developing type 2 diabetes is important for early intervention and the prevention of the current diabetes epidemic.

A number of chemical biomarkers have been proposed as indicators for the development of type 2 diabetes in women with a history of GDM. Higher fasting plasma glucose levels during pregnancy, after adjustment for BMI and age, have been shown to be predictors of the metabolic syndrome 40 months [6] and 8.5 years [7] after the index pregnancy. Similarly, we have previously shown that fasting plasma glucose levels during pregnancy and postpartum, and postpartum C-peptide and ghrelin levels, were significant risk factors for the development of type 2 diabetes in women with a previous history of GDM [8].

In the general population, several prospective studies have identified dyslipidaemia, particularly hypertriglyceridaemia, as an independent predictor of type 2 diabetes $[9,10]$. However, it is now becoming clear that many other lipid types may be useful predictors of type 2 diabetes [11, 12]. However, no studies have used plasma lipidomic analysis to identify women with a history of GDM who have an increased risk of developing type 2 diabetes. Thus, the purpose of this study was to identify lipids and lipid profiles that are predictive of the development of type 2 diabetes in a cohort of women with a previous GDM pregnancy.

\section{Methods}

Patient recruitment and sample collection The study was approved by the Mercy Health Research and Ethics Committee and written informed consent was obtained from all participants. Women were eligible for this study if they had a previous GDM pregnancy, were aged at least 18 years and English speaking. Women with pre-existing diabetes were excluded.

Between June 2003 and December 2005, 148 women were recruited during their first pregnancy diagnosed with GDM. The diagnosis of GDM was made by an OGTT at 24-28 weeks of gestation, according to the Australasian Diabetes in
Pregnancy Society (ADIPS) guidelines by either a fasting venous plasma concentration of $\geq 5.5 \mathrm{mmol} / 1$ glucose and/or $\geq 8.0 \mathrm{mmol} / 1$ glucose $2 \mathrm{~h}$ after a $75 \mathrm{~g}$ oral glucose load. All women diagnosed with GDM met with the hospital's dietitian and were advised to follow the recommended Standard of Care diet for controlling blood glucose ( $40 \%$ carbohydrate, $15 \%$ protein and $45 \%$ fat).

The women who participated in this study had an OGTT performed at 12 weeks postnatally, and only the women who had a normal glucose tolerance were further evaluated for this study. Participants were classified as having normal glucose tolerance (NGT), impaired glucose tolerance (IGT) or type 2 diabetes according to ADA criteria [13]; the criteria remained consistent for the duration of the study. At the time of the postnatal OGTT, women had their weight and height measured and a blood sample was taken for biomarker evaluation. The participants were followed up every 1-2 years for 8 10 years. During these follow-up visits, the participants underwent further OGTTs. Upon diagnosis of type 2 diabetes, the women were referred to their doctor for further specialist care.

Postnatal blood samples were collected in EDTA tubes, centrifuged at 1,000 $\mathrm{g}$ for $10 \mathrm{~min}$; plasma was supplemented with $0.1 \mathrm{mmol} / 1$ phenylmethylsulfonyl fluoride protease inhibitor (USB, Cleveland, OH, USA) and immediately stored at $-80^{\circ} \mathrm{C}$ until assayed as detailed below.

HPLC-MS analysis Plasma lipidomic analysis was performed as previously described [14]. Briefly, plasma samples were randomised prior to extraction, internal standards (stable isotope labelled and non-physiological lipid species) were added and total lipids were extracted from plasma using a single phase, chloroform:methanol extraction process. Internal standards including species within the classes of dihydroceramide (dhCer 8:0), ceramide (Cer 17:0), sphingomyelin (SM 12:0), lysophosphatidylcholine (LPC 13:0), phosphatidylcholine (PC 13:0/13:0), lysophosphatidylethanolamine (LPE 14:0), phosphatidylethanolamine (PE 17:0/17:0), phosphatidylglycerol (PG 17:0/ 17:0), phosphatidylserine (PS 17:0/17:0) and cholesterol (cholesterol $\left[d_{7}\right]$ ) were purchased from Avanti (Alabaster, AL, USA). Cholesteryl ester (CE 18:0 $\left[d_{6}\right]$ ) was purchased from CDN Isotopes (Quebec, QC, Canada). Diacylglycerol (DG 15:0/15:0) and triacylglycerol (TG 17:0/17:0/17:0) were purchased from Sigma (St Louis, MO, USA). Glucosylceramide (GluCer 16:0 $\left.\left[d_{3}\right]\right)$, lactosylceramide (LacCer 16:0 $\left.\left[d_{3}\right]\right)$ and trihexosylceramide (THC 17:0) were purchased from Matreya (Pleasant Gap, PA, USA).

Lipidomic analysis was performed using an Agilent 1200 HPLC system (Agilent Technologies, Santa Clara, CA, USA) coupled to an AB SCIEX API 4000 Q/TRAP mass spectrometer (AB Sciex, Framingham, MA, USA). Individual lipid species (300) were measured using scheduled multiple- 
reaction monitoring in positive ion mode. Lipid measures were calculated by relating the peak area of each species to the peak area of the corresponding internal standard. The measurements of 23 lipid classes were calculated by summing the individual lipid species within each class [14].

Statistical analysis Prior to association analysis, the concentration of lipid species were normalised using the interquartile range. A binary logistic regression was used to identify lipid species associated with the development of type 2 diabetes. Four traditional risk factors (age, BMI, pregnancy fasting OGTT and postnatal fasting OGTT) were used as covariates for this analysis. The lipid species associated with future diabetes with $p$ values $>0.01$ were considered as candidates for multivariate model development. Starting with traditional risk factors and $\log$ transformed values of all the candidate lipid species in a binary regression framework, lipids were sequentially removed from the model based on the most insignificant $p$ value. This process was stopped when all the lipid species in the model were significant $(p<0.05)$.

To assess the performance of the selected lipid species for diabetes prediction, a threefold stratified cross-validation training/testing framework (repeated 200 times) was employed. In each iteration, three models (risk factors, lipids, and risk factors with lipids as predictors) were trained using a logistic regression classifier. The performances of the models were assessed by measuring AUC, accuracy, specificity and sensitivity. Finally, the mean and $95 \%$ CI of the performance measures over the 600 iterations were calculated to compare the performances of models. In addition, the net reclassification improvement obtained by using the combined model compared with the risk factor only model was computed.

\section{Results}

Of the 148 women recruited to the study, 44 were excluded due to missing clinical data. During the follow-up period of 8 10 years (median 8.7 years) of the 104 participants, 77 (74\%) were NGT, six (5.8\%) developed IGT and 21 (20.2\%) developed type 2 diabetes. The median time to the development of type 2 diabetes was 8.5 years. Fifty of the 104 women were prescribed insulin during the third trimester of their pregnancy according to hospital guidelines for insulin therapy in GDM. The clinical details of the patients are presented in Table 1 . The patients who developed type 2 diabetes were significantly older and had a significantly higher BMI at the time of postnatal blood collection. In addition, they had a significantly higher pregnancy and postnatal fasting glucose concentration, and postnatal total cholesterol and triacylglycerol levels.

For all analysis, the NGT plus the IGT group was used as a reference to test the association of lipid species with the development of type 2 diabetes. The NGT and IGT participants were combined together as the IGT patients tended to fluctuate between NGT and IGT during the follow-up period. Multivariate analysis, after adjusting for age, BMI, pregnancy fasting OGTT and postnatal fasting OGTT, identified nine lipid classes and subclasses (Table 2) and 61 lipid species (electronic supplementary material [ESM Table 1]) that were significantly associated with the development of type 2 diabetes. However, after correcting for multiple comparisons, these associations were not significant. Table 3 lists the 11 lipid species that were selected for further analysis based on uncorrected $p$ values of $<0.01$. These were the ceramide species Cer 22:0 and Cer 24:0; the sphingomyelin species SM 42:1; the alkylphosphatidylcholine species $\mathrm{PC}(\mathrm{O}-36: 3)$; the

Table 1 Patient characteristics

\begin{tabular}{llll}
\hline Characteristics & $\begin{array}{l}\text { Type 2 diabetes } \\
\text { (median and IQR) } \\
(n=21)\end{array}$ & $\begin{array}{l}\text { NGT/IGT } \\
\text { (median and IQR) } \\
(n=83)\end{array}$ & $p$ value $^{\mathrm{a}}$ \\
& & & \\
\hline OGTT during pregnancy & $5.30(4.68,5.73)$ & $4.70(4.40,5.10)$ & $4.7 \times 10^{-03}$ \\
$\quad$ Fasting (mmol/l) & $10.20(9.70,11.43)$ & $9.50(8.53,10.98)$ & 0.088 \\
1 h (mmol/l) & $9.10(8.08,10.75)$ & $8.60(8.10,9.18)$ & 0.289 \\
2 h (mmol/l) & & & \\
Postnatal; time of sample collection & $35.11(31.62,37.67)$ & $31.83(29.19,35.44)$ & $1.05 \times 10^{-02}$ \\
Age (years) & $29.14(23.38,34.70)$ & $25.04(22.61,28.49)$ & $4.59 \times 10^{-02}$ \\
BMI (kg/m $\left.{ }^{2}\right)$ & $0.31(0.28,0.33)$ & $0.31(0.28,0.34)$ & 0.771 \\
Years post index & $5.00(4.78,5.32)$ & $4.60(4.40,4.90)$ & $1.49 \times 10^{-03}$ \\
Fasting OGTT (mmol/l) & $7.20(6.25,8.55)$ & $6.20(5.20,7.40)$ & $2.96 \times 10^{-02}$ \\
1 h OGTT (mmol/l) & $5.30(4.80,6.20)$ & $5.20(4.40,6.08)$ & 0.488 \\
2 h OGTT (mmol/l) & $6.00(5.03,7.12)$ & $5.20(4.63,5.95)$ & $8.25 \times 10^{-03}$ \\
Total cholesterol (mmol/l) & $1.10(0.80,1.52)$ & $0.90(0.60,1.20)$ & $3.71 \times 10^{-02}$ \\
Triacylglycerol (mmol/l) & & &
\end{tabular}

${ }^{\mathrm{a}} p$ values were computed using the Wilcoxon rank sum test

$\mathrm{IQR}$, interquartile range 
Table 2 Association of lipid classes and subclasses with future diabetes $^{\mathrm{a}}$

\begin{tabular}{|c|c|c|c|}
\hline Lipid class/subclass & $\mathrm{IQR}_{\mathrm{OR}}^{\mathrm{b}}$ & $p$ value & $p$ value $^{\mathrm{c}}$ \\
\hline Dihydroceramide & $2.03(0.97,4.27)$ & 0.064 & 0.148 \\
\hline Ceramide & $2.36(1.28,4.37)$ & $7.24 \times 10^{-03}$ & 0.082 \\
\hline Monohexosylceramide & $1.38(0.74,2.58)$ & 0.310 & 0.357 \\
\hline Dihexosylceramide & $1.49(0.77,2.88)$ & 0.244 & 0.311 \\
\hline Trihexosylceramide & $1.60(0.90,2.83)$ & 0.113 & 0.184 \\
\hline GM3 ganglioside & $1.42(0.92,2.21)$ & 0.120 & 0.184 \\
\hline Sphingomyelin & $1.95(1.02,3.75)$ & $4.70 \times 10^{-02}$ & 0.120 \\
\hline Phosphatidylcholine & $1.58(0.87,2.89)$ & 0.136 & 0.196 \\
\hline Alkylphosphatidylcholine & $2.62(1.11,6.16)$ & $2.98 \times 10^{-02}$ & 0.086 \\
\hline Alkenylphosphatidylcholine & $1.96(0.91,4.21)$ & 0.089 & 0.158 \\
\hline Lysophosphatidylcholine & $1.15(0.53,2.47)$ & 0.725 & 0.758 \\
\hline Lysoalkylphosphatidylcholine & $1.56(0.71,3.42)$ & 0.267 & 0.323 \\
\hline Phosphatidylethanolamine & $2.09(1.10,3.98)$ & $2.69 \times 10^{-02}$ & 0.086 \\
\hline Alkylphosphatidylethanolamine & $2.86(1.17,6.99)$ & $2.32 \times 10^{-02}$ & 0.086 \\
\hline Alkenylphosphatidylethanolamine & $2.22(1.16,4.27)$ & $1.85 \times 10^{-02}$ & 0.086 \\
\hline Lysophosphatidylethanolamine & $1.03(0.50,2.11)$ & 0.933 & 0.933 \\
\hline Phosphatidylinositol & $2.09(1.09,4.02)$ & $2.90 \times 10^{-02}$ & 0.086 \\
\hline Lysophosphatidylinositol & $1.49(0.78,2.84)$ & 0.235 & 0.311 \\
\hline Phosphatidylserine & $3.87(1.44,10.38)$ & $8.51 \times 10^{-03}$ & 0.082 \\
\hline Free cholesterol & $1.75(0.93,3.31)$ & 0.088 & 0.158 \\
\hline Cholesteryl ester & $2.40(1.24,4.65)$ & $1.06 \times 10^{-02}$ & 0.082 \\
\hline Diacylglycerol & $0.86(0.58,1.29)$ & 0.477 & 0.522 \\
\hline Triacylglycerol & $2.06(0.94,4.52)$ & 0.075 & 0.156 \\
\hline
\end{tabular}

${ }^{\text {a }}$ Adjusted for age, BMI, pregnancy fasting OGTT and postnatal fasting OGTT

${ }^{\mathrm{b}}$ Data are interquartile range OR $(95 \% \mathrm{CI})$

${ }^{\mathrm{c}} p$ values are corrected for multiple comparisons with the Benjamini-Hochberg method alkylphosphatidylethanolamine species $\mathrm{PE}(\mathrm{O}-36: 3)$ and the alkenylphosphatidylethanolamine species $\mathrm{PE}(\mathrm{P}-36: 2)$; the

Table 3 Association of lipid species with future diabetes ${ }^{\mathrm{a}}$

\begin{tabular}{llll}
\hline Lipid species & IQR OR $^{\mathrm{b}}$ & $p$ value & $p$ value $^{\mathrm{c}}$ \\
\hline Cer 22:0 & $2.61(1.34,5.09)$ & $6.00 \times 10^{-03}$ & 0.221 \\
Cer 24:0 & $2.54(1.34,4.79)$ & $4.96 \times 10^{-03}$ & 0.221 \\
SM 42:1 & $3.01(1.37,6.62)$ & $7.43 \times 10^{-03}$ & 0.221 \\
PC(O-36:3) & $2.91(1.33,6.35)$ & $8.84 \times 10^{-03}$ & 0.221 \\
PE(O-36:3) & $3.63(1.50,8.78)$ & $5.27 \times 10^{-03}$ & 0.221 \\
PE(P-36:2) & $5.44(2.10,14.11)$ & $7.42 \times 10^{-04}$ & 0.221 \\
PI 36:1 & $2.74(1.30,5.75)$ & $9.17 \times 10^{-03}$ & 0.221 \\
PS 38:4 & $4.05(1.67,9.82)$ & $2.53 \times 10^{-03}$ & 0.221 \\
CE 18:1 & $2.62(1.39,4.95)$ & $3.67 \times 10^{-03}$ & 0.221 \\
CE 18:2 & $3.40(1.48,7.82)$ & $4.83 \times 10^{-03}$ & 0.221 \\
CE 20:4 & $2.52(1.28,4.98)$ & $8.99 \times 10^{-03}$ & 0.221 \\
\hline
\end{tabular}

${ }^{\text {a }}$ Adjusted for age, BMI, pregnancy fasting OGTT and postnatal fasting OGTT

${ }^{\mathrm{b}}$ Data are interquartile range $\mathrm{OR}(95 \% \mathrm{CI})$

${ }^{\mathrm{c}} p$ values were corrected for multiple comparisons with the BenjaminiHochberg method phosphatidylinositol species PI 36:1; the phosphatidylserine species PS 38:4; and the cholesteryl ester species CE 18:1, CE 18:2 and CE 20:4, which were all positively associated with the development of type 2 diabetes.

A logistic regression model was used to assess the association of these 11 lipid species combined (identified in Table 3) with the development of type 2 diabetes (Table 4). Using log transformed data with a sequential elimination approach, three lipid species remained significantly and positively associated

Table 4 Final logistic regression model

\begin{tabular}{lrrrl}
\hline Model term & $\beta$ coefficient & \multicolumn{1}{l}{ SE } & $t$ value & $p$ value \\
\hline Intercept & -90.511 & 17.116 & -5.288 & $7.75 \times 10^{-07}$ \\
Age & 0.201 & 0.060 & 3.353 & $1.1 \times 10^{-03}$ \\
BMI & 0.093 & 0.056 & 1.662 & 0.099 \\
Pregnancy fasting OGTT & 1.566 & 0.458 & 3.420 & $9.20 \times 10^{-04}$ \\
Postnatal fasting OGTT & -0.002 & 0.785 & -0.002 & 0.998 \\
PE(P-36:2) & 8.626 & 2.255 & 3.826 & $2.32 \times 10^{-04}$ \\
PS 38:4 & 7.255 & 1.885 & 3.849 & $2.14 \times 10^{-04}$ \\
CE 20:4 & 6.132 & 2.304 & 2.662 & $9.10 \times 10^{-03}$ \\
\hline
\end{tabular}


with the development of type 2 diabetes: CE 20:4, PE(P-36:2) and PS 38:4.

These three lipid species were then used in a predictive model for the development of type 2 diabetes (Table 5). Six risk factors (age, BMI, and levels of pregnancy fasting glucose, postnatal fasting glucose, triacylglycerol and total cholesterol) were used in the base model. The addition of the three lipid species CE 20:4, PE(P-36:2) and PS 38:4 to the base model improved the accuracy $(80.5 \%-83.6 \%)$, increased the area under the receiver operating characteristic (ROC) curve (0.756-0.865) (Fig. 1), increased its discriminatory ability (increase in sensitivity from $34.3 \%-59.0 \%$ ) and resulted in a net reclassification index of $22.3 \%$.

\section{Discussion}

In our cohort of women with previous GDM, we found that age, BMI and levels of pregnancy fasting glucose, postnatal fasting glucose, total cholesterol and triacylglycerol were significantly higher in women who went on to develop type 2 diabetes than those who did not (NGT or IGT). Thus, we created a clinical risk model for the development of type 2 diabetes using these six risk factors. Importantly, the inclusion of three plasma lipid species to this base model improved the prediction of type 2 diabetes in women with a previous GDM pregnancy; the combination of six clinical risk factors and three lipid species, CE 20:4, PE(P-36:2) and PS 38:4, produced a model with increased discriminatory ability (an increase in sensitivity of $15.7 \%$ [34.3\%-59.0\%]). Although there was a concomitant decrease in specificity of $2.3 \%$ $(92.2 \%-89.9 \%)$, clinically this decrease would result in only a small increase in false positives, and overall we observed a net reclassification index of $22.3 \%$. The addition of the lipid species into the model resulted in the total cholesterol and triacylglycerol variables losing significance, indicating collinearity between the clinical lipid measures and our lipid species. Furthermore, the final model demonstrates that the individual lipid species are better predictors than the clinical lipid measures.
Only one prior study has profiled plasma lipid species in women with a previous history of GDM [15]. In this study, 100 serum samples from the Hyperglycemia and Pregnancy Outcome (HAPO) study (mean postnatal follow-up of 22 months) were analysed. Participants were stratified by glucose tolerance during a previous index pregnancy into three risk groups, overt GDM, those with glucose values in the upper quartile but below GDM levels, and controls. Substantial differences in metabolite profiles were apparent between the two at-risk groups and controls, particularly in concentrations of phospholipids (four metabolites), acylcarnitines (three metabolites), short- and long-chain fatty acids (three metabolites) and diacylglycerols (four metabolites). However, none of these metabolites matched the lipid species identified in the current study and no prediction model was created.

Several reports have investigated the additive effectiveness of including individual lipid species for the prediction of diabetes $[11,12,16-18]$. In the Australian Diabetes, Obesity and Lifestyle (AusDiab) study, the inclusion of 21 individual plasma lipid species to triacylglycerol and $\mathrm{HbA}_{1 \mathrm{c}}$ as predictors in the diabetes risk classification model resulted in a statistically significant gain in area under the ROC curve and a significant net reclassification improvement [12]. Interestingly, ceramide and its biosynthetic precursor dihydroceramide are consistently associated with the development of diabetes $[12,16]$. In the current study, total ceramide and ceramide lipid species Cer 22:0 and Cer 24:0 were positively associated with the development of type 2 diabetes (prior to correction for multiple comparisons). We also found that the ceramide metabolite sphingomyelin SM 42:1 was a significant predictor of type 2 diabetes, although also not retained in the final model. Collectively, these findings suggest that the ceramide biosynthetic pathway is a common metabolic process in the pathogenesis of type 2 diabetes in both the general population and in women with previous GDM.

Ceramide is recognised as a proinflammatory lipid, although the mechanistic basis of the observed elevated plasma ceramide level in type 2 diabetes is not fully defined. LDL enriched in ceramide has been shown to lead to increased insulin resistance in skeletal muscle and a proinflammatory state in macrophages [19]. IL-6 is thought to be involved in the metabolic link between ceramide, insulin resistance and inflammation [20].
Table 5 Classification performance of models for future diabetes

\begin{tabular}{lccc}
\hline Performance measure & Risk factors & Lipids & Lipids+risk factor model \\
\hline No. of features & 6 & 3 & 9 \\
Accuracy & $80.5(80.1,80.9)$ & $81.8(81.4,82.1)$ & $83.6(83.2,84.1)$ \\
Area under the ROC curve & $0.756(0.748,0.763)$ & $0.845(0.839,0.850)$ & $0.865(0.859,0.871)$ \\
Sensitivity & $34.3(32.9,35.6)$ & $39.8(38.4,41.1)$ & $59.0(57.4,60.5)$ \\
Specificity & $92.2(91.8,92.6)$ & $92.4(92.0,92.8)$ & $89.9(89.4,90.4)$ \\
Net reclassification index & & & $22.3 \%(20.6 \%, 24.1 \%)$ \\
\hline
\end{tabular}

Data represent average values from 3-fold cross validation, 200 repeats $(95 \% \mathrm{CI})$

${ }^{\mathrm{a}}$ Net reclassification improvement relative to the risk factors model 


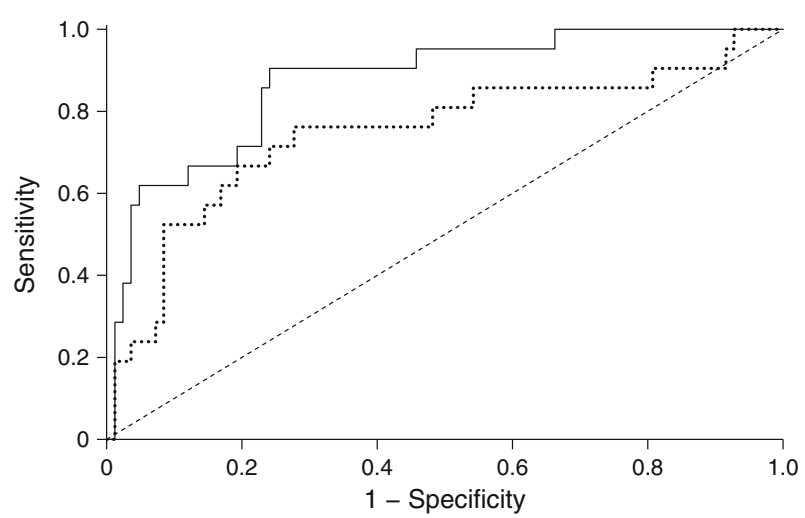

Fig. 1 ROC curves for prediction of type 2 diabetes. Performance of the models to predict type 2 diabetes was evaluated within a threefold crossvalidation framework (repeated 200 times). Average true positive and false-positive rates were then used to generate the ROC curves. The dotted line denotes the risk factor only model that contained six clinical risk factors as features, including age, BMI, and levels of pregnancy fasting glucose, postnatal fasting glucose, triacylglycerol and total cholesterol. This model attains an average AUC of 0.756 . The solid line represents the performance of the model developed using the six previously described risk factors and three lipids, PE(P-36:2), PS 38:4 and CE 20:4, as features. This combined model achieves an average AUC of 0.865

Furthermore, the accumulation of ceramide has been demonstrated to be detrimental to pancreatic beta cells [21-23] and may, therefore, play a direct role in the pathogenesis of type 2 diabetes. Alkenylphosphatidylethanolamines (plasmalogens) contain a vinyl ether linkage that is particularly susceptible to oxidation by reactive oxygen species, and as such they are proposed to play an antioxidant role in membranes and lipoproteins [24]. Ethanolamine plasmalogens prevent oxidation of cholesterol by reducing its oxidisability in phospholipid bilayers [25] and are involved in the regulation of cholesterol esterification and efflux [26]. We have previously observed that some plasmalogen species are negatively associated with conditions of high oxidative stress, including type 2 diabetes [16] and coronary artery disease [27]. The observed positive association of plasmalogens with future type 2 diabetes in this cohort may therefore represent an early compensatory mechanism in response to elevated oxidative stress in these individuals.

Also worth noting is that while total cholesterol is not considered a risk factor for type 2 diabetes in the general population, total cholesteryl esters and individual cholesteryl ester species have been shown to be significant predictors of type 2 diabetes $[12,14]$. Similarly, in this study, total cholesteryl ester and several cholesteryl ester lipid species were positively associated with the development of type 2 diabetes. Although no other studies have profiled cholesteryl esters as predictors of diabetes in women with previous GDM, a prospective case-control study identified $35 \%$ of women with GDM as 'high-risk' for the development of diabetes 10 years after diagnosis based on cardiometabolic risk measurements in pregnancy that included LDL and HDL [28].
Interestingly, our previous studies in two populationbased cohorts also found that CE 20:4 was associated with the development of type 2 diabetes. Specifically, in the AusDiab and San Antonio Family Heart Study (SAFHS) [16], CE 20:4 was significantly and positively associated with type 2 diabetes and impaired glucose tolerance and/or impaired fasting glucose in a cross-sectional analysis. In the same study, neither PE(P-36:2) nor PS 38:4 showed a significant association with type 2 diabetes, indicating that changes preceding type 2 diabetes are not necessarily reflected in the disease state. These initial changes may relate to early compensatory mechanisms that are subsequently overcome as the disease progresses.

There are limitations of the current study. First, recruitment of participants was ad hoc and did not include all women diagnosed with GDM during the study period. Second, we could not correct for family history and ethnicity, which are known risk factors for diabetes, as we did not have this data available. Nevertheless, women attending this hospital comprise a mixed population including European, Asian, Middle Eastern and African ethnicities. Another limitation of our study is its relatively small sample size, which might have led to falsepositive associations or overestimation of the effect size for the individual lipids. Moreover, true associations might have been missed and the effect size might have been underestimated for some of the lipid species. Although we have used a stringent cross-validation approach to assess the performance of the classification models, we were unable to provide an independent validation of the predictive model.

Women with a previous history of GDM have a substantially increased risk of developing type 2 diabetes later in life [5]. Thus, the identification of women at increased risk provides an opportunity for early treatment to prevent onset or progression of the disease. Indeed, lifestyle intervention and/or medications have been shown to delay the development of type 2 diabetes in women with a history of GDM [29, 30]. During a median follow-up of 30 months, troglitazone treatment was associated with a $55 \%$ reduction in the development of type 2 diabetes in women previously diagnosed with GDM [30]. Furthermore, in women with a history of GDM, 3 years after both intensive lifestyle and metformin therapy, the incidence of diabetes was reduced by approximately $50 \%$ compared with the placebo group [29].

In conclusion, the inclusion of three lipid species to a riskfactor base model increased its predictive ability to identify women with a previous GDM pregnancy at risk of developing type 2 diabetes. Early identification of women at greatest risk may assist in improved targeted interventions aimed at the prevention of type 2 diabetes following a GDM pregnancy. 
Evaluation using a larger study population and a longer follow-up period is needed to confirm these results.

Acknowledgements This manuscript is dedicated to the memory of Emeritus Professor Norman Albert Beischer AO - an inspiring leader, obstetrician, educator, researcher, mentor and philanthropist.

C.C.J. Louzios, A. Bohren and S. Ristevski (Department of Obstetrics and Gynaecology, University of Melbourne, Heidelberg, VIC, Australia) are thanked for their assistance with patient recruitment.

Funding ML is supported by a Career Development Fellowship from the National Health and Medical Research Council (NHMRC) of Australia (grant no. 1047025). PJM is supported by a NHMRC Senior Research Fellowship (grant no. 1042095). The work described in this manuscript was funded by the Medical Research Foundation for Women and Babies (ML, HMG), NHMRC of Australia (PJM) and the OIS Program of the Victorian Government, Australia (PJM).

Duality of interest The authors declare that there is no duality of interest associated with this manuscript.

Contribution statement ML conceived the study and carried out the experiments. All authors contributed to the study design and procedures. DJ recruited the patients. PAM, GW, KH and PJM analysed the data. ML, PAM, HMG and PJM drafted the manuscript. All authors revised the manuscript critically for important intellectual content and approved the final manuscript. ML and PJM are the guarantors of this work.

\section{References}

1. Shaw JE, Sicree RA, Zimmet PZ (2010) Global estimates of the prevalence of diabetes for 2010 and 2030. Diabetes Res Clin Pract 87:4-14

2. Roglic G, Unwin N, Bennett PH et al (2005) The burden of mortality attributable to diabetes: realistic estimates for the year 2000 . Diabetes Care 28:2130-2135

3. Chen L, Magliano DJ, Zimmet PZ (2012) The worldwide epidemiology of type 2 diabetes mellitus - present and future perspectives. Nat Rev Endocrinol 8:228-236

4. Hossain P, Kawar B, El Nahas M (2007) Obesity and diabetes in the developing world - a growing challenge. N Engl J Med 356:213-215

5. Lee AJ, Hiscock RJ, Wein P, Walker SP, Permezel M (2007) Gestational diabetes mellitus: clinical predictors and long-term risk of developing type 2 diabetes: a retrospective cohort study using survival analysis. Diabetes Care 30:878-883

6. Akinci B, Celtik A, Yener S, Yesil S (2010) Prediction of developing metabolic syndrome after gestational diabetes mellitus. Fertil Steril 93:1248-1254

7. Bo S, Monge L, Macchetta C et al (2004) Prior gestational hyperglycemia: a long-term predictor of the metabolic syndrome. J Endocrinol Investig 27:629-635

8. Lappas M, Jinks D, Ugoni A, Louizos CC, Permezel M, Georgiou HM (2014) Postpartum plasma C-peptide and ghrelin concentrations are predictive of type 2 diabetes in women with previous gestational diabetes mellitus. J Diabetes. doi:10.1111/1753-0407. 12209

9. Bonora E, Targher G, Alberiche M et al (2002) Predictors of insulin sensitivity in type 2 diabetes mellitus. Diabet Med 19:535-542

10. Joseph J, Svartberg J, Njolstad I, Schirmer H (2011) Risk factors for type 2 diabetes in groups stratified according to metabolic syndrome: a 10-year follow-up of the Tromso Study. Eur J Epidemiol $26: 117-124$
11. Floegel A, Stefan N, Yu Z et al (2013) Identification of serum metabolites associated with risk of type 2 diabetes using a targeted metabolomic approach. Diabetes 62:639-648

12. Wong G, Barlow CK, Weir JM et al (2013) Inclusion of plasma lipid species improves classification of individuals at risk of type 2 diabetes. PLOS ONE 8:e76577

13. Genuth S, Alberti KG, Bennett P et al (2003) Follow-up report on the diagnosis of diabetes mellitus. Diabetes Care 26:3160-3167

14. Weir JM, Wong G, Barlow CK et al (2013) Plasma lipid profiling in a large population-based cohort. J Lipid Res 54:2898-2908

15. Anderson SG, Dunn WB, Banerjee M et al (2014) Evidence that multiple defects in lipid regulation occur before hyperglycemia during the prodrome of type-2 diabetes. PLOS ONE 9:e103217

16. Meikle PJ, Wong G, Barlow CK et al (2013) Plasma lipid profiling shows similar associations with prediabetes and type 2 diabetes. PLOS ONE 8:e74341

17. Meikle PJ, Wong G, Barlow CK, Kingwell BA (2014) Lipidomics: potential role in risk prediction and therapeutic monitoring for diabetes and cardiovascular disease. Pharmacol Ther 143:12-23

18. Rhee EP, Cheng S, Larson MG et al (2011) Lipid profiling identifies a triacylglycerol signature of insulin resistance and improves diabetes prediction in humans. J Clin Invest 121:1402-1411

19. Boon J, Hoy AJ, Stark R et al (2013) Ceramides contained in LDL are elevated in type 2 diabetes and promote inflammation and skeletal muscle insulin resistance. Diabetes 62:401-410

20. de Mello VD, Lankinen M, Schwab U et al (2009) Link between plasma ceramides, inflammation and insulin resistance: association with serum IL-6 concentration in patients with coronary heart disease. Diabetologia 52:2612-2615

21. Boslem E, MacIntosh G, Preston AM et al (2011) A lipidomic screen of palmitate-treated MIN6 beta-cells links sphingolipid metabolites with endoplasmic reticulum (ER) stress and impaired protein trafficking. Biochem J 435:267-276

22. Manukyan L, Ubhayasekera SJ, Bergquist J, Sargsyan E, Bergsten P (2015) Palmitate-induced impairments of beta-cell function are linked with generation of specific ceramide species via acylation of sphingosine. Endocrinology 156:802-812

23. Boslem E, Meikle PJ, Biden TJ (2012) Roles of ceramide and sphingolipids in pancreatic beta-cell function and dysfunction. Islets 4:177-187

24. Lessig J, Fuchs B (2009) Plasmalogens in biological systems: their role in oxidative processes in biological membranes, their contribution to pathological processes and aging and plasmalogen analysis. Curr Med Chem 16:2021-2041

25. Maeba R, Ueta N (2003) Ethanolamine plasmalogens prevent the oxidation of cholesterol by reducing the oxidizability of cholesterol in phospholipid bilayers. J Lipid Res 44:164-171

26. Mankidy R, Ahiahonu PW, Ma H et al (2010) Membrane plasmalogen composition and cellular cholesterol regulation: a structure activity study. Lipids Health Dis 9:62

27. Meikle PJ, Wong G, Tsorotes D et al (2011) Plasma lipidomic analysis of stable and unstable coronary artery disease. Arterioscler Thromb Vasc Biol 31:2723-2732

28. Barden A, Singh R, Walters B, Phillips M, Beilin LJ (2013) A simple scoring method using cardiometabolic risk measurements in pregnancy to determine 10-year risk of type 2 diabetes in women with gestational diabetes. Nutr Diabetes 3, e72

29. Ratner RE, Christophi CA, Metzger BE et al (2008) Prevention of diabetes in women with a history of gestational diabetes: effects of metformin and lifestyle interventions. J Clin Endocrinol Metab 93: 4774-4779

30. Buchanan TA, Xiang AH, Peters RK et al (2002) Preservation of pancreatic beta-cell function and prevention of type 2 diabetes by pharmacological treatment of insulin resistance in high-risk Hispanic women. Diabetes 51:2796-2803 